\title{
SPECTRAL SPECKLE FILTER FOR SAR IMAGERY
}

\author{
Gintautas Palubinskas \\ German Aerospace Center DLR \\ Remote Sensing Technology Institute \\ Oberpfaffenhofen, 82234 Wessling, Germany \\ Gintautas.Palubinskas@dlr.de
}

\begin{abstract}
Despite of a lot of efforts to develop a good de-speckling method for radar/SAR imagery (see e.g. [1, 2]) since 80 's, this topic is still actual nowadays. Recently several pathbased de-noising methods were proposed [3-6] which represent a state of the art in this field. In this paper a new de-speckling method, resulting from a signal processing viewpoint, is presented and compared to some of the methods mentioned above.
\end{abstract}

Index Terms - De-speckling, SAR, frequency decomposition, edge thresholding

\section{INTRODUCTION}

Speckle is a multiplicative noise and is usually observed in radar/SAR imagery. Its presence causes serious difficulties for an automatic and quantitative analysis of SAR data. Since several decades it engages a lot of scientists working towards de-speckling of such data. Nevertheless this topic is still actual nowadays. Recently several path-based denoising methods were proposed [3-6] which represent a state of the art in this field. First, Buades proposed a nonlocal means de-noising method to remove an additive noise [3]. Then, Deledalle has extended this approach to a multiplicative noise case [4]. Another variant of non-local means filter is proposed in [5]. Similar ideas are followed in parallel in a selective means filter which is mainly based on the analysis of a coefficient of variation [6]. Still it is quite difficult to find a good compromise between a smoothing power and preservation of very fine details, complexity and intuitive usage. In this paper a new de-speckling method, resulting from a signal processing viewpoint on the problem, is presented and compared to some of the methods mentioned above.

\section{GENERAL FILTER FRAMEWORK}

Almost all de-noising methods can be seen as a realization of the following general filter framework consisting mainly of the three following parts.

\subsection{Window size selection}

In order to remove/suppress a noise one should perform some operation (usually averaging) in some neighborhood (usually square window). The window size defines how much noise is removed.

\subsection{Pixel selection in a window (where to filter?)}

Of course simple averaging in a window will distort fine geometrical features such as points, lines and so on. In order to preserve such features averaging should be performed not on all pixels or otherwise only on selected pixels in a window. Generally speaking only similar pixels should be used for averaging. For pixel similarity various criteria can be used e.g. weak edge or gradient, weak texture, same distribution (pdf) and so on.

\subsection{Definition of weight values (how much to filter?)}

Once the pixels used in a filtering are determined there remains only a last task to be solved: are all pixels of the same importance (equal weights) or should they be differently weighted in an average. These weights can be derived from distance measure (e.g. Gaussian filter) or generally speaking from a similarity measure between pixels (e.g. calculated in the previous step).

\section{SPECTRAL SPECKLE FILTER SSF}

In this section a new de-speckling method of SAR image using spectral speckle filter (SSF) is proposed (see Figure 1).

First, SAR image is transformed into a spectral domain using Fourier transform where filters much more precisely can be defined. The resultant spectrum is decomposed into low and high frequency parts using corresponding low and high pass filters. Parameter of the low pass filter (LPF) cut-off frequency - controls the window size used for filtering. The high frequency spectrum part is transformed back into signal domain resulting in a high frequency (edge 
or gradient) image. Low frequencies corresponding to speckle are thresholded using speckle statistics (e.g. estimated in a homogeneous region) and suppressed using appropriate weighting. This step covers the two last steps of the general filter framework. Finally, weighted high frequency image is combined with a remaining low frequency spectrum part transformed back into signal domain. This last operation delivers a de-speckled SAR image.

\section{EXPERIMENTS}

In this section a comparison of three de-speckling methods:

- Non-local means filter (NLM) [3], [4]

- Selective means filter (SM) [6]

- Spectral speckle filter (SSF)

is presented for TerraSAR-X high resolution Spotlight image of Munich city (famous October Fest location). For a visual interpretation see results in Figure 3. NLM result looks most smooth while still preserving main structures. It tends to distort very fine structures and over-smoothing is observed in very bright regions. SM performs practically no smoothing in bright regions whereas smoothing works well in dark regions. SSF preserves well very small bright targets e.g. in the forest, but smoothing amount is less in homogeneous regions. Ratio images of noisy image to denoised image: $n=\frac{i}{\hat{i}}$ for all three methods are presented in Figure 2. Ratio image ideally represents only speckle thus no structures should be seen. In our case still some structures can be recognized, maybe in NLM result in less extent.

Quantitative quality is evaluated using several known measures: equivalent number of looks

$$
E N L=\frac{E(i)^{2}}{\operatorname{Var}(i)},
$$

Relative bias (mean percentage error)

$$
\text { Bias }=\sum \frac{\hat{i}-i}{i},
$$

Mean values $E\{n\}$ and variances $\operatorname{Var}\{n\}$ estimated on ratio images. De-speckling is working well, when the Bias is below $20 \%$ [7], $E\{n\}$ is about 1 and $\operatorname{Var}\{n\}$ is about $1 / L$. The results for a selected homogeneous region (blue box in Figure 3) in TerraSAR-X high resolution Spotlight image of Munich city are presented in Table 1 . The used SAR data exhibited an equivalent number of looks $\mathrm{L}=1.36$.

By analyzing ENL values we see that the largest amount of filtering exhibits NML method closely followed by SM. The largest Bias value is observed for NLM whereas for other two methods it is under $20 \%$. NML and SSF exhibit similarly good $E\{n\}$ values and SSF the best $\operatorname{Var}\{n\}$ value.
NML method was run with defaults parameters [4] whereas SM was very difficult to configure due a lot of adjustable parameters. SFF result is influenced only by two parameters: cut-off frequency of LPF - amount of smoothing (or equivalently a window size in signal domain) and threshold for finding low edges (edges caused by speckle). Thus latter parameter depends mainly on speckle statistics.

Table 1. Comparison of different de-speckling filters for a selected homogeneous region in TerraSAR-X image ( $\mathrm{L}=1.36)$.

\begin{tabular}{|l|l|l|l|l|}
\hline Filter & ENL & $\begin{array}{l}\text { Bias } \\
<20 \%\end{array}$ & $\begin{array}{l}\mathrm{E}\{\mathrm{n}\} \\
=1 \text { (ideal) }\end{array}$ & $\begin{array}{l}\text { Var }\{\mathrm{n}\}=1 / \mathrm{L} \\
=0.74 \text { (ideal) }\end{array}$ \\
\hline None & 1.14 & - & - & - \\
\hline NLM & $\mathbf{1 5 . 7}$ & $29.6 \%$ & $\mathbf{0 . 9 2}$ & 0.42 \\
\hline SM & 13.3 & $\mathbf{1 0 . 4} \%$ & 1.27 & 0.96 \\
\hline SSF & 5.7 & $19.7 \%$ & 1.09 & $\mathbf{0 . 5 9}$ \\
\hline
\end{tabular}

\section{CONCLUSIONS}

A new de-speckling method for SAR imagery is proposed which is based on Fourier transform and suppression of edge information caused by speckle. Visual inspection of results show that the proposed method is capable to preserve very fine structures in SAR image, e.g. in forest, and thus it can be interesting as a pre-processing option for target detection applications. Quantitative quality evaluation of the proposed method and comparison with some other state of the art methods is performed calculating several quality measures such as equivalent number of looks, relative bias and statistics on ratio images. Further investigation can be directed towards better theoretical foundation of the proposed method and automatic data-driven estimation of unknown parameters.

\section{ACKNOWLEGMENT}

TerraSAR-X data were provided by DLR through the Science Project MTH0948.

\section{REFERENCES}

[1] J.-S. Lee, "Speckle supression and analysis for synthetic aperture radar images," Opt. Eng., 25(5), pp. 636-643, 1986. 
[2] M. Walessa, and M. Datcu, "Model-based despeckling and information extraction from SAR images," IEEE Transactions on Geoscience and Remote Sensing, 38(5), pp. 2258-2269, 2000.

[3] A. Buades, B. Coll, and J. Morel, "A non-local algorithm for image denoising," Proc. IEEE Computer Vision and Pattern Recognition, vol. 2, pp. 60-65, 2005.

[4] C.A. Deledalle, L. Denis, and F. Tupin, "Iterative weighted Maximum Likelihood denoising with probabilistic patch-based weights," IEEE Transactions on Image Processing, 18(12), pp. 2661-2672, 2009.
[5] K. Dabov, A. Foi, V. Katkovnik, and K. Egiazarian, "Image denoising by sparse 3D transform-domain collaborative filtering," IEEE Transactions on Image Processing, 16(8), pp. 2080-2095, 2007.

[6] Th. Esch, A. Schenk, G. Palubinskas, J. Hoffmann, A. Roth and S. Dech, "Spatially adaptive and radiometrically selective removal of speckle in SAR data based on analysis of multiplicative noise," Unpublished manuscript, 2011.

[7] S. Solbøa, and T. Eltoft, "A Stationary Wavelet-Domain Wiener Filter for Correlated Speckle," IEEE Transactions on Geoscience and Remote Sensing, 46(4), pp. 1219-1230, 2008.

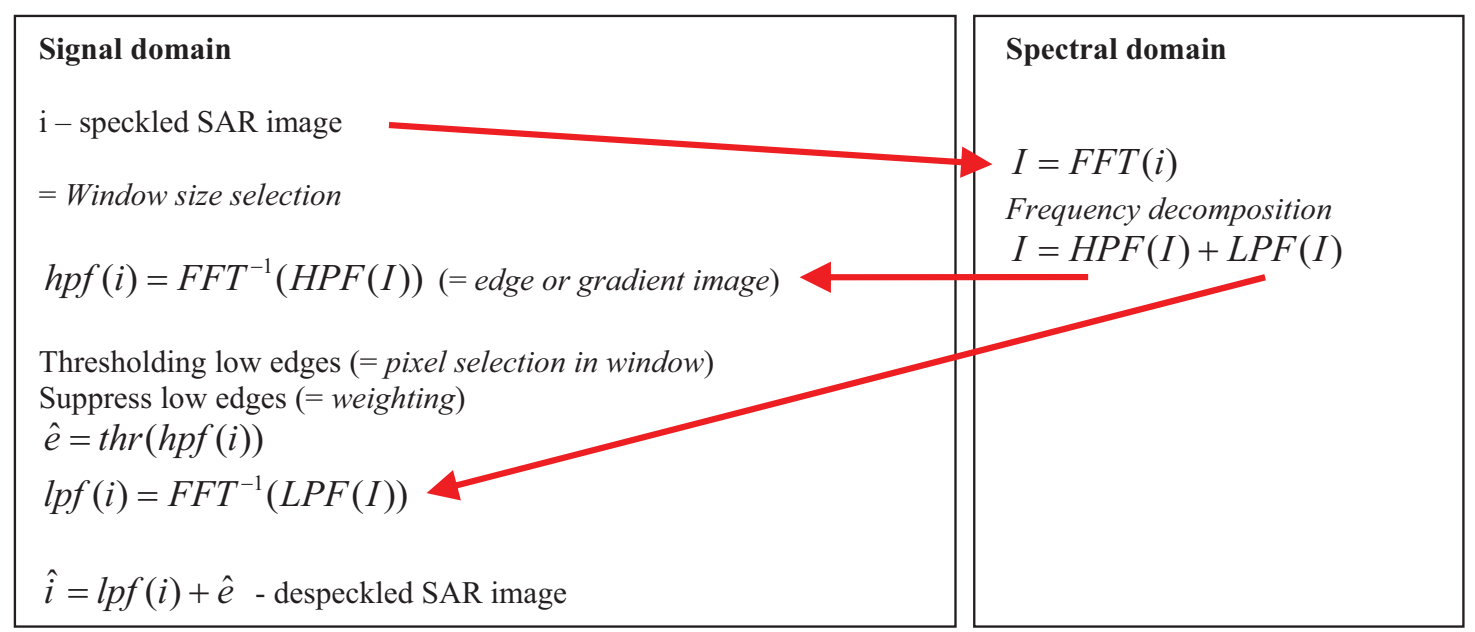

Figure 1. Description of spectral speckle filter SSF for SAR imagery de-speckling.

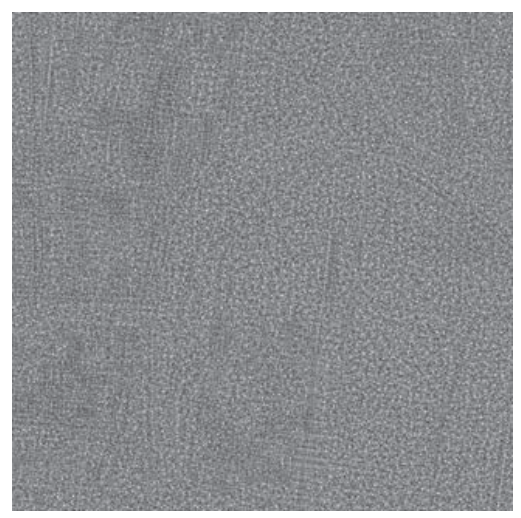

NLM

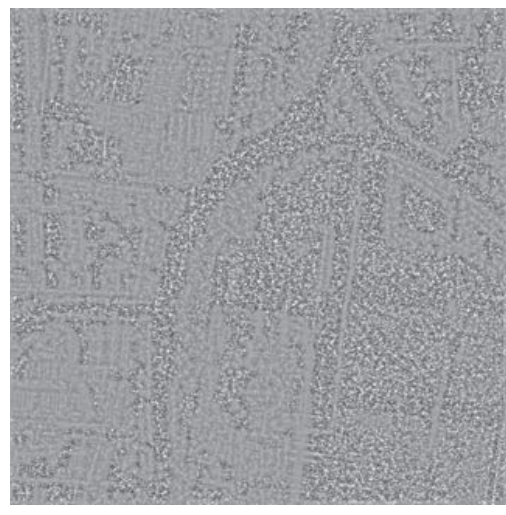

SM

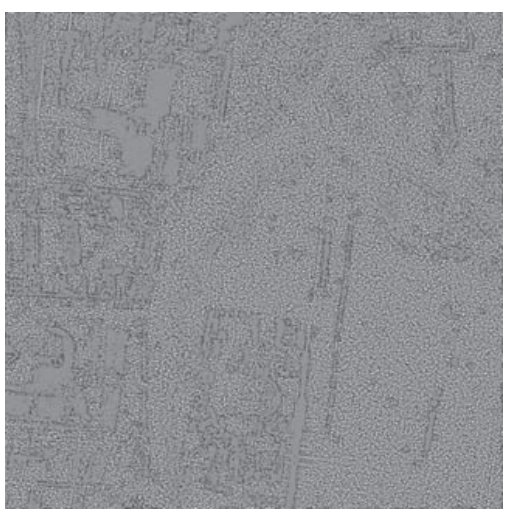

SSF

Figure 2. Comparison of ratio images for three de-speckling methods on a high resolution TerraSAR-X Spotlight image. 


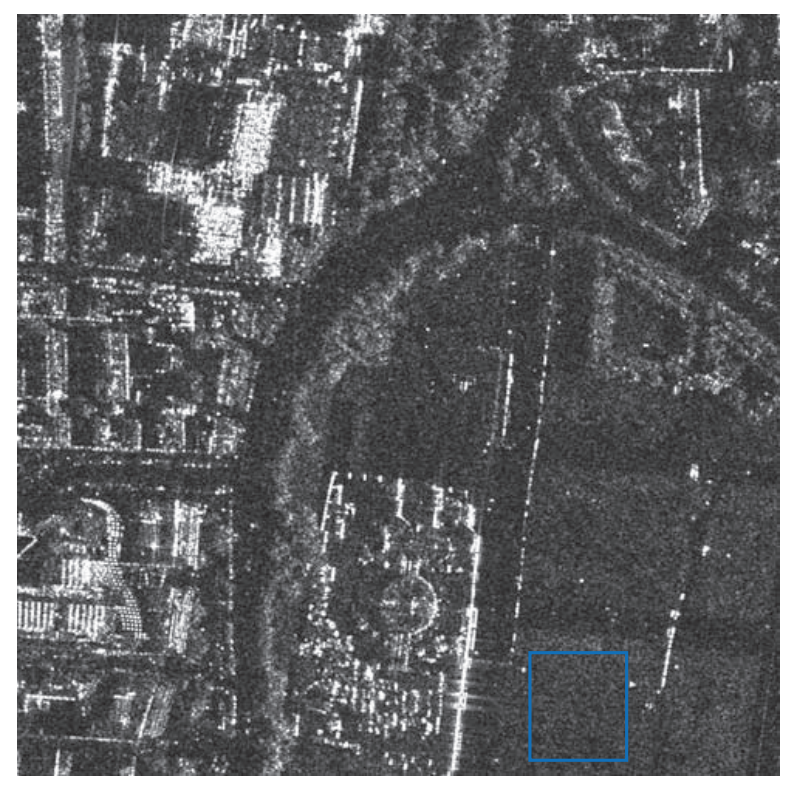

Original

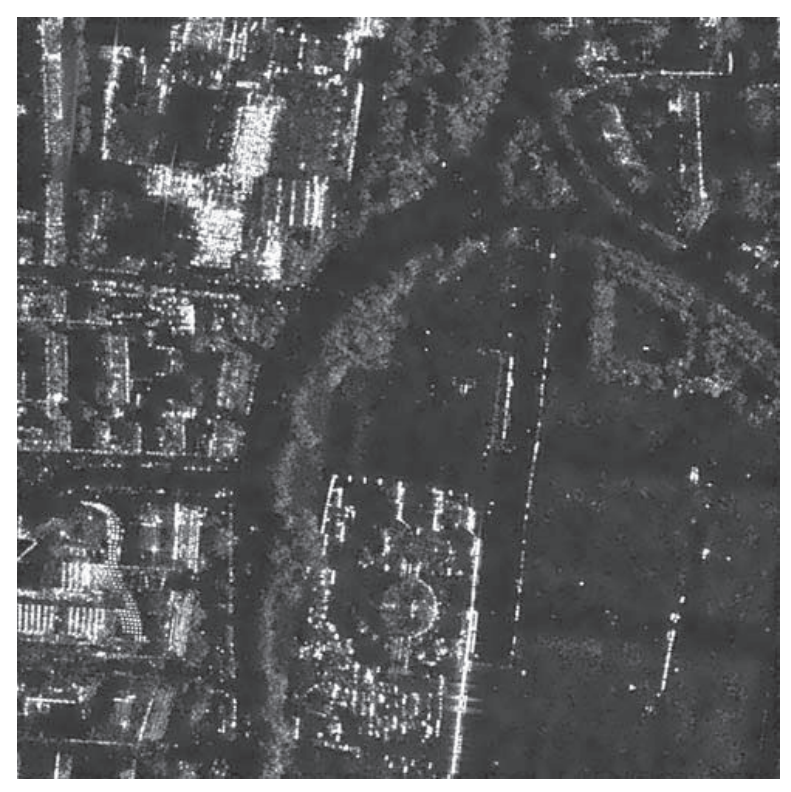

$\mathrm{SM}$

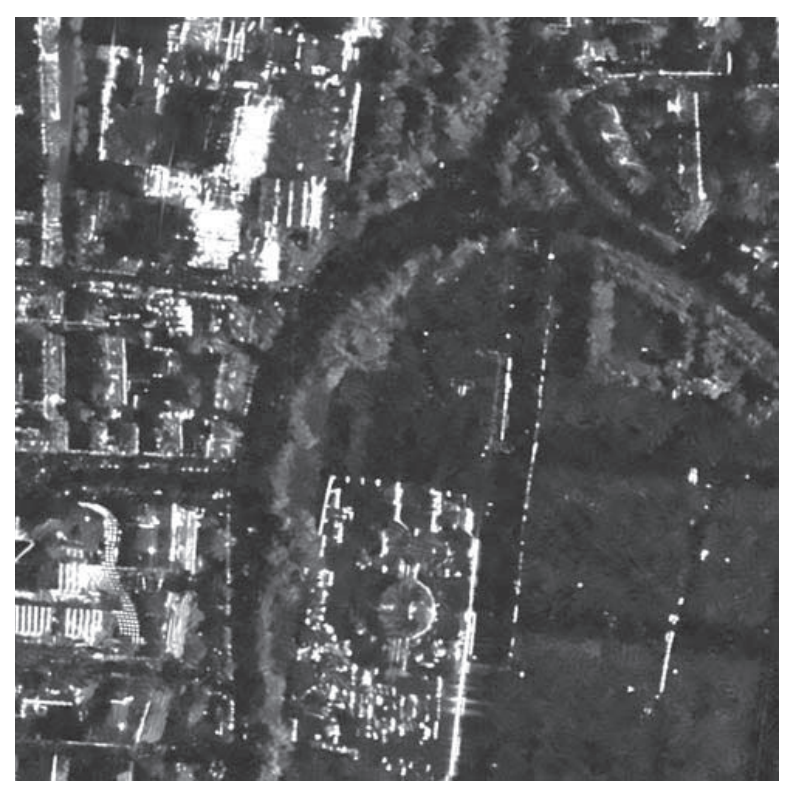

NLM

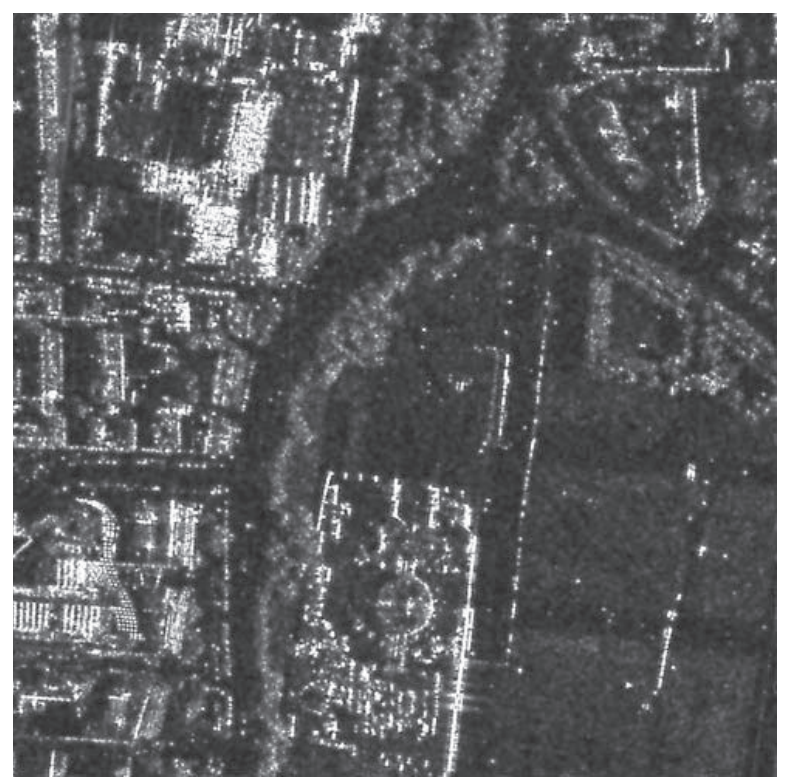

SSF

Figure 3. Comparison of three de-speckling methods on a high resolution TerraSAR-X Spotlight image (blue box - homogeneous region). 